\title{
Understanding Hepatic Encephalopathy
}

Nicolas WEISS 1,2,3, MD, PhD, Rajiv JALAN 4, MD, PhD, and Dominique THABUT 1, 2, 5, MD, PhD.

1 Brain Liver Pitié-Salpêtrière (BLIPS) Study Group, Groupement Hospitalier PitiéSalpêtrière-Charles Foix, Assistance Publique - Hôpitaux de Paris, France

2 INSERM UMR_S 938, CDR Saint-Antoine Maladies métaboliques, biliaires et fibroinflammatoire du foie \& Institut de Cardiométabolisme et Nutrition, ICAN, Paris, France

3 Unité de réanimation neurologique, Département de neurologie, pôle des maladies du système nerveux, Groupement Hospitalier Pitié-Salpêtrière-Charles Foix, Assistance Publique - Hôpitaux de Paris et Institut de neurosciences translationnelles IHU-A-ICM, Paris, France.

4 Liver Failure Group, UCL Institute for Liver and Digestive Health, UCL Medical School, Royal Free Hospital, London, UK

5 Unité de Soins Intensifs d'Hépato-gastroentérologie, Groupement Hospitalier PitiéSalpêtrière-Charles Foix, Assistance Publique - Hôpitaux de Paris et Université Pierre et Marie Curie Paris 6, Paris, France.

\section{Correspondance:}

Nicolas WEISS

Unité de réanimation neurologique,

Fédération de neurologie, pôle des maladies du système nerveux,

Groupement Hospitalier Pitié-Salpêtrière-Charles Foix,

Assistance Publique - Hôpitaux de Paris,

47-83 boulevard de l'Hôpital, 75013 Paris, France

Tel : +331421627 70

Mail : nicolas.weiss@aphp.fr 
Hepatic encephalopathy (HE) is defined as a neurological or a neuropsychological complication caused by liver disease or portosystemic shunting. The clinical spectrum is highly variable ranging from mild neuropsychological disturbances to coma [1]. Depending upon the underlying liver disease, HE is classified into 3 types; type A, secondary to acute liver failure (ALF); type B, secondary to portosystemic shunting and type C, secondary to cirrhosis in the presence or not of shunting [2]. The most recent human and animal data confirmed the previously supposed role of hyperammonemia, but also outlines the role of associated factors like inflammation in the development of HE.

The main source of ammonia ( $\mathrm{NH} 4+)$ in the portal system has long be thought to be intestinal bacterial production explaining the use of non-absorbable disaccharides (e.g. lactulose) and non-absorbable antibiotics (e.g. rifaximin) [3]. Recent data shows however that the main source is glutamine catabolism by glutaminase in the gut [3]. Thus, polymorphisms in the gene coding for glutaminase predicts the development of HE in cirrhotic patients [4]. In the systemic circulation, $\mathrm{NH} 4+$ is increased due to reduced urea cycle enzyme activity that occurs in liver failure and/or portosystemic shunting. This increased amount of $\mathrm{NH} 4+$ is converted to glutamine in muscle cells and in astrocytes, through the action of glutamine synthetase $[3,5]$. These latter abnormalities both explain why sarcopenia represents a factor that makes patients susceptible to develop HE in cirrhosis and the development of brain edema patients with ALF and cirrhosis. Only, recently directly targeting hyperammonemia by using ammonia lowering agents have been proposed (e.g. glycerol-phenylbutyrate, Lornithine-L-aspartate). According to its osmotic potential, the acute intracytoplasmic increase of glutamine in ALF is responsible for cytotoxic edema affecting the astrocytes and for vasogenic edema when the blood-brain barrier (BBB) is altered 
$[3,5]$. In cirrhosis, the glutamine increase is gradual and astrocytes respond by progressively extruding intracytoplasmic myoinositol and taurine to try and maintain osmotic equilibrium. This largely explains why brain edema is rarely present in cirrhosis and/or in acute on chronic liver failure (ACLF) $[6,7]$. In neurons, glutamine is deaminated into glutamate, the most important excitatory neurotransmitter in the brain, that stimulates neurons [8]. This could account for anxious behaviour, agitation or seizures in ALF. In contrast, in cirrhosis, compensatory mechanisms are responsible for a decreased expression of both glutamate carriers (GLT-1) and glutamate post-synaptic receptors that explains slowing, sleepiness and altered consciousness.

Amino acid imbalance has been hypothesised to participate in HE physiopathology $[5,9]$. Cerebral levels of aromatic aminoacids (AAA) are increased due to altered liver function, increased amount of free tryptophan due to hypoalbuminemia but also to altered transport through the BBB. As a consequence, there is an imbalance in the synthesis of dopamine, norepinephrine, serotonine and in the synthesis of 'false neurotransmitters', octopamine or tyramine $[3,5,8]$. Other pathophysiological mechanisms such as cerebral energy failure associated to hyperammonemia, altered immune responses, reduced blood flow, mitochondrial dysfunction and inhibition of alpha-ketoglutarate dehydrogenase, a rate-limiting tricarboxylic acid cycle enzyme have been described [9].

Whereas $\mathrm{NH} 4+$ is not directly correlated with neurological status, several studies demonstrated a good correlation with the presence of systemic inflammatory response syndrome (SIRS) and the blood amount of TNF-alpha or IL-6 [10]. Sepsis and systemic inflammation are a hallmark of the severity of cirrhosis and infection is a classical triggering event of HE [11]. Outside the field of cirrhosis, encephalopathy 
related to sepsis has been described. Modification of the intestinal microbiota is emerging as a major factor associated with $\mathrm{HE}[5,12]$. It modulation has been proposed to explain the effect of lactulose and rifaximin with conflicting results. More recently, fecal transplantation has been proposed as a therapeutic strategy in HE. Liver failure is associated with altered intestinal barrier function, which is responsible for bacterial translocation and activation of innate immune system. Nevertheless, this innate immune response is altered in liver failure as defined by altered neutrophil phagocytic capacity, reduced reticuloendothelial system and reduced hepatic synthesis of antimicrobial proteins [13]. ACLF is characterised by upregulation of proinflammatory cytokines (IL-1, IL-6, IL-17, TNF-alpha, IFN-gamma) compared to stable cirrhotic patients [14]. In the brain of HE patients, astrocytes and microglial cells respond to systemic inflammation by producing IL-1beta and IL-6 that stimulate adhesion of neutrophils and their trans-endothelial migration through the BBB, the release of chemokines, proteases and reactive oxygene species [5]. As a result, microglial cells present an activated phenotype. Current data suggest that the brain of patients with cirrhosis are sensitised to the effect of systemic inflammation and infection. Therefore, prompt treatment of any infection is an important therapeutic intervention. Noteworthy, anti-inflammatory treatments, i.e. indomethacin or ibuprofen, blocking microglial activation, are able to prevent both neurocognitive symptoms and brain edema in several animal models of HE [10].

Apart from hyperammonemia and inflammation, other factors are suspected to be involved in the physiopathology of HE. Thus, neurotransmission is largely impaired in $\mathrm{HE}$, either as a consequence, as previously discussed for both glutamate, dopamine and serotonin, or as a cause, increased intracerebral levels of benzodiazepine-like compounds or neurosteroids that end in an increased gamma-amino-butyric acid 
(GABA) tone [8]. The presence of benzodiazepine-like compounds explains why the use of flumazenil had been proposed in the treatment of HE. Due to liver failure, several substances have been reported in excess in HE patients: phenols, mercaptans, short chain fatty acids, manganese or bile acids [9].

Using metabolomics, we recently showed that HE patients displayed several drugs, especially antimicrobial agents (eg. metronidazole, fluconazole or betalactamins), in their CSF [9]. This is in line with clinical studies that could link neurological symptoms with beta-lactams or proton pumps inhibitor intake in cirrhotic patients [15]. Remarkably, these drugs are all substrates of ATP-binding cassette (ABC)transporters expressed on the BBB and responsible for efflux of several drugs outside the brain. We recently showed in HE animal models that ABC-transporters expression was altered compared to cirrhotic animals without HE (personal data). These data suggest that some neurological abnormalities could be related to druginduced encephalopathy. Nevertheless, recent animal data showed that both bilirubin and bile acids exposure could induce a down-regulation of P-glycoprotein (P-gp), the main $A B C$-transporter, on the BBB and that this decreased expression could favour cerebral GABA concentrations. Administration of these drugs should be carefully considered in cirrhotic patients and considered in the differential diagnosis of brain dysfunction.

Recent progress in cellular biology and immunology has modified our concept of HE physiopathology and will, in the future, provide new treatments. 


\section{References}

1. Mouri S, Tripon S, Rudler M, et al. FOUR score, a reliable score for assessing overt hepatic encephalopathy in cirrhotic patients. Neurocrit Care. 2015;22:251257.

2. Ferenci P, Lockwood A, Mullen K, Tarter R, Weissenborn K, Blei AT. Hepatic encephalopathy--definition, nomenclature, diagnosis, and quantification: final report of the working party at the 11th World Congresses of Gastroenterology, Vienna, 1998. Hepatol Baltim Md. 2002;35:716-721.

3. Holecek M. Ammonia and amino acid profiles in liver cirrhosis: effects of variables leading to hepatic encephalopathy. Nutr Burbank Los Angel Cty Calif. 2015;31:14-20.

4. Romero-Gómez M, Jover M, Del Campo JA, et al. Variations in the promoter region of the glutaminase gene and the development of hepatic encephalopathy in patients with cirrhosis: a cohort study. Ann Intern Med. 2010;153:281-288.

5. Romero-Gómez M, Montagnese S, Jalan R. Hepatic encephalopathy in patients with acute decompensation of cirrhosis and acute-on-chronic liver failure. $\mathrm{J}$ Hepatol. 2015;62:437-447.

6. Weiss N, Rosselli M, Mouri S, et al. Modification in CSF specific gravity in acutely decompensated cirrhosis and acute on chronic liver failure independent of encephalopathy, evidences for an early blood-CSF barrier dysfunction in cirrhosis. Metab Brain Dis. Epub 2016 Oct 11.

7. Selvapatt N, Singanayagam A, Wendon J, Antoniades CG. Understanding infection susceptibility in patients with acute-on-chronic liver failure. Intensive Care Med. 2014;40:1363-1366.

8. Palomero-Gallagher N, Zilles K. Neurotransmitter receptor alterations in hepatic encephalopathy: a review. Arch Biochem Biophys. 2013;536:109-121.

9. Weiss N, Barbier Saint Hilaire P, Colsch B, et al. Cerebrospinal fluid metabolomics highlights dysregulation of energy metabolism in overt hepatic encephalopathy. J Hepatol. 2016;65:1120-1130.

10. Shawcross DL, Davies NA, Williams R, Jalan R. Systemic inflammatory response exacerbates the neuropsychological effects of induced hyperammonemia in cirrhosis. J Hepatol. 2004;40:247-254.

11. Thabut D, Massard J, Gangloff A, et al. Model for end-stage liver disease score and systemic inflammatory response are major prognostic factors in patients with cirrhosis and acute functional renal failure. Hepatol Baltim Md. 2007;46:1872-1882.

12. Bajaj JS, O'Leary JG, Reddy KR, et al. Second infections independently increase mortality in hospitalized patients with cirrhosis: the North American 
consortium for the study of end-stage liver disease (NACSELD) experience. Hepatol Baltim Md. 2012;56:2328-2335.

13. Wasmuth HE, Kunz D, Yagmur E, et al. Patients with acute on chronic liver failure display "sepsis-like" immune paralysis. J Hepatol. 2005;42:195-201.

14. Berry PA, Antoniades CG, Carey I, et al. Severity of the compensatory antiinflammatory response determined by monocyte HLA-DR expression may assist outcome prediction in cirrhosis. Intensive Care Med. 2011;37:453-460.

15. Weiss N, Junot C, Rudler M, et al. Hepatic vs. drug-induced encephalopathy in cirrhotic patients? Liver Int Off J Int Assoc Study Liver. 2016;36:1233-1234.

\section{Figure: Hepatic encephalopathy physiopathology hallmarks: ammonia (1, 2, 3 and 4 ) and inflammation (a, b and c) are the main actors.}

1, As opposed to previous theories, the main source of $\mathrm{NH}^{+}$in the portal circulation is intestinal catabolism of glutamine; 2, Once increased in the portal circulation, $\mathrm{NH} 4+$ increases in the systemic circulation due to liver failure (reduced activity of urea cycle enzymes) and/or portosystemic shunting; 3 , In the case of liver failure, $\mathrm{NH}^{+}$detoxification into glutamine through glutamine synthetase is only possible in the muscle cells and the astrocytes. In muscles cells, this reaction needs the use of BCAA, which are decreased in cirrhosis. In astrocytes, the rapid increase in glutamine explains the occurrence of cytotoxic edema seen in the astrocytes through its osmotic potential. In more progressive disease, osmotic components, myoinositol and taurine, are extruded outside the cytoplasm to counterbalance the glutamine increase to try and prevent astrocytic edema. 4, The observed neuronal effects may vary; hyperstimulation and seizures may be due to accumulation of glutamate in the synaptic cleft; coma due to increased GABA in the brain; psychomotor disturbance due to other neurotransmitters.

a, Due to modification of the gut microbiota, intestinal barrier function is altered and bacterial translocation abnormally increased. This leads to an increase of pro-inflammatory cytokines in the portal circulation; b. Acquired cellular immune depression related to liver disease further favours proinflammatory cytokine production; c, Systemic inflammation induces an alteration of blood-brain barrier permeability, and pro-inflammatory cytokines, especially IL-1 $\beta$ and IL-6, activate both astrocytes and microglial cells. Several of these steps can be worsened by hyperammonemia, as shown in vitro on cell cultures or in vivo in animal models.

Other abnormalities have been described in HE physiopathology. Among them, accumulation of several substances such as increased AAA levels, increased GABA tone through benzodiazepine-like components or neurosteroids. Recent studies implicated drug accumulation in the cerebrospinal fluid as contributing to the physiopathology of HE.

Different treatment strategies that has been validated or proposed in the treatment of HE are represented preceded by the pill icon in the figure.

Abbreviations: $A A A$, aromatic amino acids; $A B C$ transporters, ATP-binding cassette transporters; $B C A A$, branched-chain amino acids; $C D$, cluster of differentiation; GABA, gamma-amino-butyric acid ; GLN, glutamine; HE, hepatic encephalopathy; HLA-DR, human leukocyte antigen - antigen D related; IFN, interferon; IL, interleukine; LPS, lipopolysaccharide; NH4+, ammonia; TNF, tumor necrosis factor. 


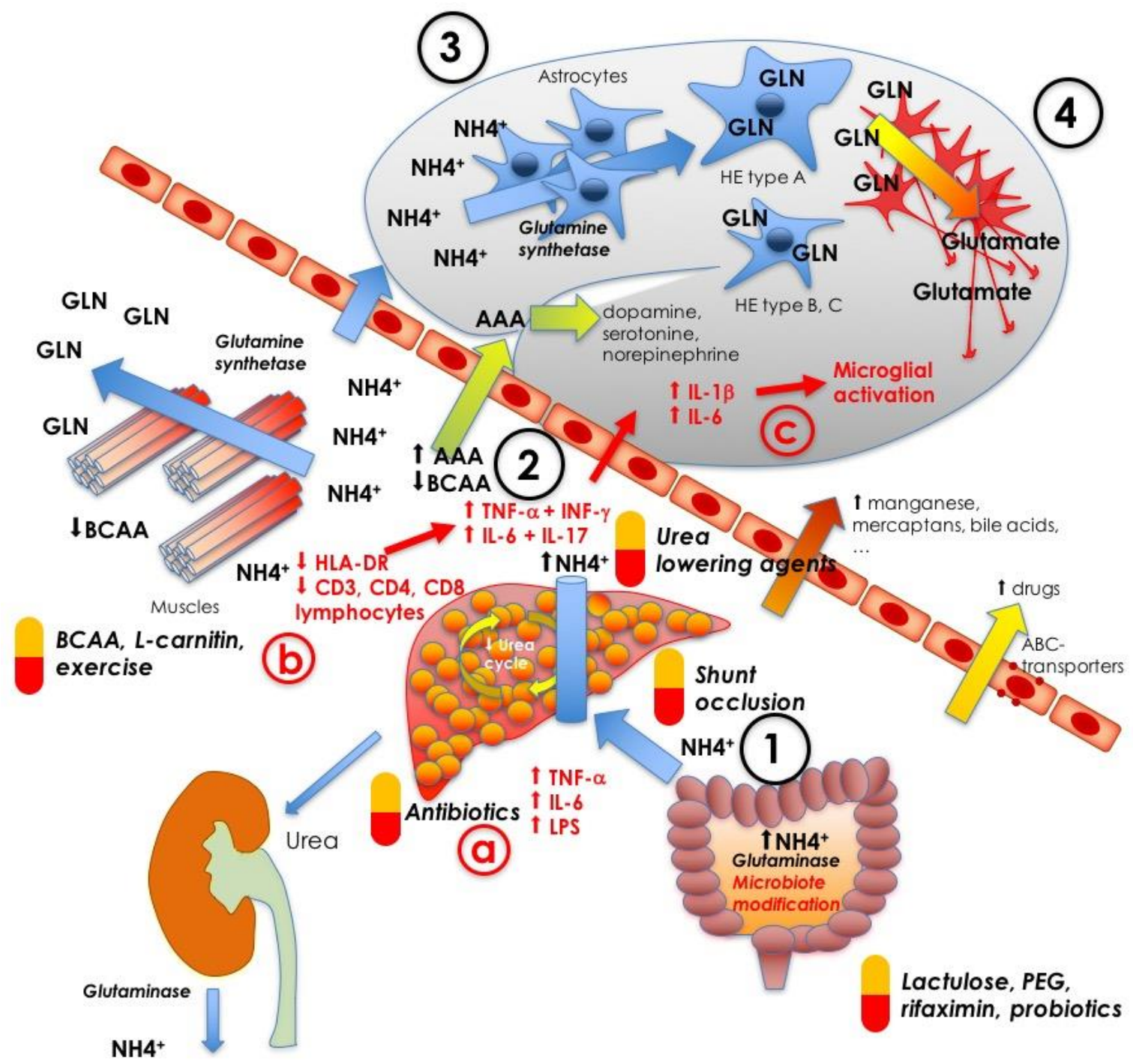

